\title{
Fast Modeling System of Digital Plant for Virtual Education
}

 \\ Beijing Research Center for Information Technology in Agriculture, Beijing,100097, China \\ aemail: guoxy@nercita.org.cn
}

Keywords: Virtual Education; Digital Plant; Modeling system

\begin{abstract}
Virtual education is widely used in many fields including agricultural training and popular science. Modeling technologies and software tools for content making of virtual educations about digital plant is shortages and it is an obvious limitation of relevant applications. We present a fast modeling technology and developed a system of digital plant for virtual education. The method depends on real geometric data of plant collected by three-dimensional device, and a template-based modeling algorithm is employed for rapidly reconstruction of complex plant organs. The system consists of several modules including data processing, knowledge management, geometric modeling, graphics support and user interface. The system supports users to make education content about digital plant. Finally, some typical applications are given and some future works are discussed.
\end{abstract}

\section{Introduction}

Virtual Reality is becoming an effective way for modern education in many fields such as technological training, popular science, callan learning [1-4]. It has particular advantage compared with traditional education method. Virtual education has attracted many researchers and it provided a new paradigm for imparting knowledge. Petrakou [5] presented a framework of interacting through avatars: virtual worlds as a context for online education. Ieronutti and Chittaro [6] provided a method employing virtual humans for education and training in X3D/VRML worlds. I. Munene [7] carried out work about experimenting in distance education. John et al. [8] discussed virtual education in universities as a technological imperative. Duan and Zhu [9] implement a real time interaction in virtual education system based on VRML. Nowadays, E-Learning has become the latest trend in the training and development industry profit from usage of network technology [10].

Virtual education has brought a large requirement in digital content in different fields and applications. In agriculture training and education, media content as well as relevant software tools is lack for virtual environment. Digital plant is an adaptive solution for this shortage. In past decades, digital plant is a rising research issue in agriculture and botany [11]. It takes a crucial part in typical applications aiming at agricultural technological training. Many researchers proposed various methods to resolve problems in digital plant or virtual plant [12]. However, the presented approaches are not directly suitable for applications of virtual educations with both three-dimensional plant models and agricultural knowledge. To provide a technology and software tool for virtual education, we propose a fast modeling technology and develop an accessorial modeling system in this paper.

\section{Education Resource And 3D Models Acquisition}

Resource and three-dimensional models are main content of virtual education. Here resource includes plant bionomics knowledge, agricultural production technologies and popular science. The three-dimensional original data of plant shape is the most important work for modeling. 3D digitizer and laser scanner can be used for data acquisition. For assigned plants, its shape can be measured by $3 \mathrm{D}$ device and recorded as point cloud or feature points. For instance, we got point cloud model of a maize plant including all stalks and leaves by use of 3D scanner FastSCAN, as shown in Fig.1. The original data could be processed by algorithm of filter to construct a template models for modeling. 


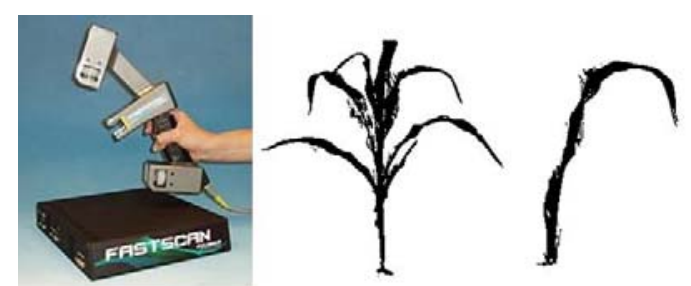

Fig.1. 3D Laser Scanner FastSCAN and a 3D Scanned Point Cloud Model of Maize

\section{Fast Modeling of Digital Plant}

\section{Parameterized Expression of Plant Structures}

Parameterization and parameterized modeling method are effective way for construction of virtual plant shape [13]. We presented a parameterized expression of plant structures. For a given plant organ, parameters are used to construct the main shape and constraint. For example, we can define a set of parameters to describe a maize plant, including leaf length, leaf width, azimuth, and so on. Under the parameter, the three-dimensional models can be constructed by a serious of mathematical equations and matrix transform. Eq.(1) describe a translation and rotation process by matrix respectively. However, parameterized technology is not good for complex plant structure such as leaf drapes, thus other approaches should be used such as template-based method.

$$
V^{\prime}=\left[\begin{array}{c}
V_{x}^{\prime} \\
V_{x}^{\prime} \\
V_{y}^{\prime} \\
1
\end{array}\right]=\text { Trans } \cdot V=\left[\begin{array}{cccc}
1 & 0 & 0 & \operatorname{Trans}_{x} \\
0 & 1 & 0 & \operatorname{Trans}_{y} \\
0 & 0 & 1 & \operatorname{Trans}_{z} \\
0 & 0 & 0 & 1
\end{array}\right] \cdot\left[\begin{array}{c}
V_{x} \\
V_{y} \\
V_{z} \\
1
\end{array}\right]
$$

$$
V^{\prime}=\left[\begin{array}{c}
V_{x}{ }^{\prime} \\
V_{y}{ }^{\prime} \\
V_{z}{ }^{\prime} \\
1
\end{array}\right]=\operatorname{Rot}_{\text {direction }} \cdot V=\left[\begin{array}{cccc}
\cos (\theta) & -\sin (\theta) & 0 & 0 \\
\sin (\theta) & \cos (\theta) & 0 & 0 \\
0 & 0 & 1 & 0 \\
0 & 0 & 0 & 1
\end{array}\right] \cdot\left[\begin{array}{c}
V_{x} \\
V_{y} \\
V_{z} \\
1
\end{array}\right]
$$

\section{Template-based Modeling of Complex Plant Organs}

It is a hard task to describe 3D structure by a set of parameters. Modeling of complex plant organs such as leaves is still a challenge both in computer graphics and agriculture. We present a modeling algorithm based on template, which take a set of well processed complex organs' 3D model as examples. For a new plant models modeling, a group of template models are selected under a serious constrains and then they are deformed by control of key parameters to construct the plant even canopy models. Template-based modeling process was described in instance [14]. The morphing process can be implemented by a set of affine transform.

\section{Interactive Adjustment of Plant Frame Form}

General modeling tools for virtual plant only provide construction way for models. It is a hard problem to change the models shape in general tools by a set of fussy adjustment on vertexes. The main difference between plants can be recognized in two aspects: topology and details of organ. To implement fast and convenient changing way for plant models' editing, we present an interactive adjustment mode for plant frame form, beyond adjustment on vertexes for organ details. The editing way allows directly changing in three-dimensional space by I/O devices. The interactive mode can accelerate design and promote modeling efficiency.

\section{Function Modules And System Development}
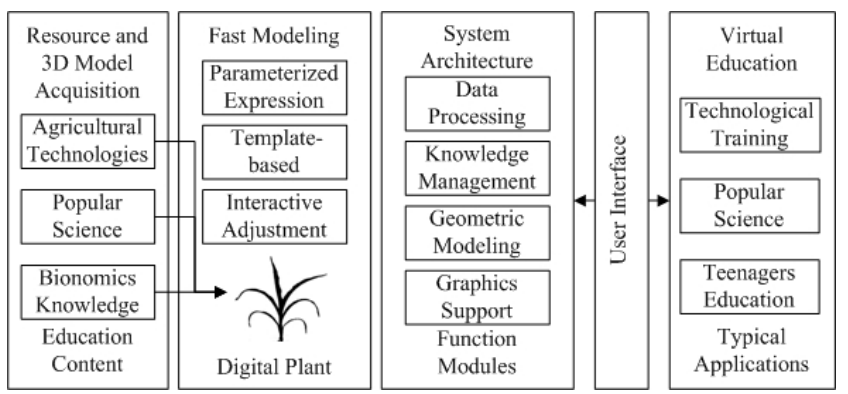

Fig.2. The flowchart of this work and the System Architecture

\section{System Architecture}

To implement presented algorithm, we developed a fast modeling system for digital plant. The 
system is developed by $\mathrm{C}++$ program language and OpenGL graphic library [15]. The architecture of system is shown in Fig. 2 including resource acquisition, fast modeling, developments and applications layers. The development is carried out by a core kernel of digital plant.

\section{Function Modules}

The modeling system includes four functional modules: data processing, knowledge management, geometric modeling and graphics support. The modules are controlled by interface elements by users to implement virtual education and typical applications, as shown in Fig.5. The modules are introduced as follow.Data Processing: This module provides three-dimensional data processing function foe digital plant models. To construct various plant models, the templates should be changed and edited. The module supported users to adjust the models and then implemented data processing about relevant changing. The module included all operations interactive adjustment which is introduced in past sections. Knowledge Management: Knowledge is the kernel core of virtual education, thus the knowledge management is the most important task of the system. The module managed all information and knowledge content relevant to typical or pointed education, where all knowledge was organized in an information database including plant bionomics knowledge, agricultural production technologies and popular science. Geometric Modeling: This module consist all geometric modeling algorithm and implementation of mathematical equations. The methods including parameterized expression and template-based modeling which were introduced in past sections are implemented in this module. Three-dimensional digital plant models are constructed by this module by a serious of mathematical matrix or equations. Graphics Support: The graphics module support drawing of graphic elements such as point, line, polygon and solid, as well as realistic rendering, including texture mapping, lighting simulation and material setting. The digital plant models which were constructed in geometric modeling were drawn and rendered by this module. The module also provided interactive ports to user interface and geometric module.

\section{Knowledge Relating}

The definition of knowledge includes plant bionomics knowledge, agricultural production technologies and popular science, and the plant bionomics knowledge in growth is important for digital model construction. We collected crucial information such as key date of growth and development of organ of stalks, leaves, flowers and construct a database to support modeling. The growth data were related onto digital plant models to control the geometric shape according a natural rule of plant. On the other hand, some internal properties of plant are also considered as knowledge, such as S-curve in plant lift period. Knowledge combined to models was defined as related properties of digital plant, and it could be directly recalled and displayed under the requirement of applications such as virtual education.

\section{Typical Applications for Virtual Education}

The modeling method as well as system can be used for digital plant reconstruction and simulations, which can be extended and support applications in virtual educations. Fig.3 (a) shows a knowledge explaining application about lighting distribution in maize canopy, the interface allows user to learn lighting radiation translation process and calculate simulation result interactively. Fig.3 (b) gives a simulation scene of maize production by automatic tractor for virtual training of agricultural technologies. The intuitionistic and interesting interface and interacting mode make virtual education for teenager easy.

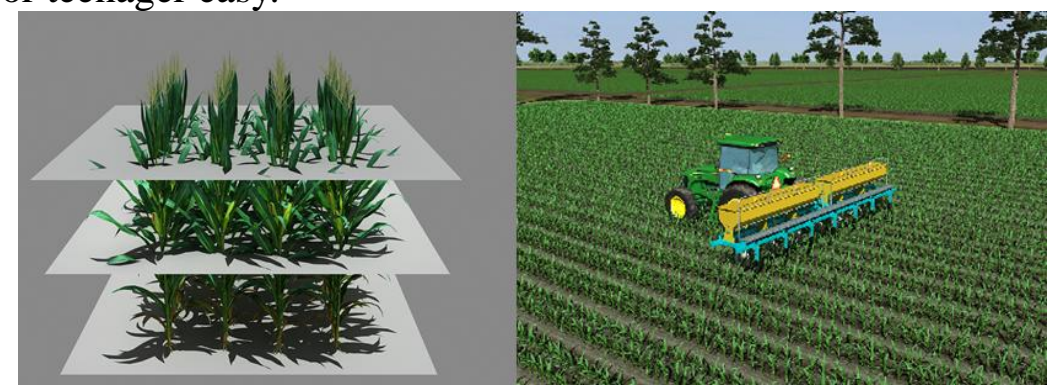

Fig.3. Typical applications of the proposed method and system 


\section{Conclusions}

To sum up, a fast modeling approach of digital plant for virtual education was proposed, and a modeling system was developed as a tool. The modeling algorithm is designed for digital plant modeling to construct accurate three-dimensional geometric shape models of plant. Parameterized expression and template-based modeling methods were employed, and the system also provided interactive adjustment mode for plant frame form. The system provided interactive interface for users supported by several kernel modules including data processing, knowledge management, fast modeling, graphics support. The main function of the system is to provide education content about digital plant in many typical applications such as technological training, public science and teenagers learning. It is a direct and efficient software tool for virtual education. In future, we focus on integration of more education knowledge and construction of large three-dimensional digital plant model database.

\section{Acknowledgement}

This work is supported by National Science \& Technology Pillar Program (Grant No. 2012BAD35B01); by Special Fund for S\&T Innovation of Beijing Academy of Agriculture and Forestry Sciences (Grant No. KJCX201204007).

\section{References}

[1] W. Ma, Y. Zhong, S. Tso, et al. A hierarchically structured and constraint-based data model for intuitive and precise solid modeling in a virtual reality environment. Computer-Aided

Design,2004,36 (10):903-928.

[2] N. Seymour, A. Gallagher, S. Roman, et al. Virtual Reality Training Improves Operating Room Performance. Annals of Surgery, 2002, 236(4): 458-464.

[3] G. Farrell. The Development of Virtual Education: A global perspective. The Commonwealth of Learning Press, Canada ,1999.

[4] H. Kan, V. Duffy, C. Su. An Internet virtual reality collaborative environment for effective product design. Computers in Industry, 2002;45(2):197-213.

[5] Alexandra Petrakou. Interacting through avatars: Virtual worlds as a context for online education. Computers \& Education, 2010, 54(4):1020-1027.

[6] L. Ieronutti, L. Chittaro. Employing virtual humans for education and training in X3D/VRML worlds. Computers \& Education, 2007, 49(1):93-109.

[7] I. Munene. Experimenting in distance education: The African virtual university (AVU) and the paradox of the World Bank in Kenya-A rejoinder. International Journal of Educational Development,2007,27(1):77-85.

[8] O. John, S. Gurmak, D. Lisa. Virtual education in universities: a technological imperative.

British Journal of Educational Technology, 2001,32(5):511-523.

[9] X. Duan, J. Zhu. The implementation of real time interaction in virtual education system based on VRML. Proc. of 4th international conference on virtual reality and its applications in industry, 2004,539-542.

[10]T. Welsh, R. Wanberg, G. Brown, et al. E-learning: emerging uses, empirical results and future directions. International Journal of Training and Development, 2003, 7(4):245-258.

[11]C. Zhao, S. Lu, X. Guo, et al. Exploration of Digital Plant and Its Technology System. Scientia Agricultura Sinica, 2010,43(10):2023-2030.

[12]B. Oborny, C. Mony, T. Herben. From virtual plants to real communities: A review of modelling clonal growth. Ecological Modelling, 2012,234(1):3-19.

[13]F. Anastacio, P. Prusinkiewicz, M. Sousa. Sketch-based parameterization of L-systems using illustration-inspired construction lines and depth modulation. Computers \& Graphics, 2009,33(4): 440-451.

[14]B. Xiao, X. Guo, X. Du, et al. An interactive digital design system for corn modeling. Mathematical and Computer Modelling, 2010, 51(11-12):1383-1389.

[15] Shreiner,D.,M. Woo,J.Neider and T.Davis, OpenGL Programming Guide: the official guide to learning OpenGL, Addison-Wesly,2004. 